\title{
The Attitudes about Complex Therapy Scale (ACTS) in Type 2 Diabetes and Cardiovascular Disease: Development, Validity and Reliability
}

Rebecca J Stack ${ }^{1 *}$, Peter R Noyce ${ }^{2}$, John P New ${ }^{3}$, Martin Gibson ${ }^{3}$, Rachel A Elliott ${ }^{4}$, Chris Bundy ${ }^{5}$

${ }^{1}$ Division of Psychology, Nottingham Trent University, Nottingham, England.

${ }^{2}$ School of Pharmacy and Pharmaceutical Sciences, University of Manchester, England.

${ }^{3}$ Salford Royal Foundation Trust, Salford, England.

${ }^{4}$ School of Pharmacy, University of Nottingham, England

${ }^{5}$ Manchester Academic Health Science Centre, University of Manchester, England.

\section{Abstract}

Background: Type 2 diabetes is associated with cardiovascular disease, and patients with both conditions are prescribed complex medication regimens.

Aim: The aim was to develop a reliable and valid measure of attitudes associated with the prescription and management of multiple medicines in patients with Type 2 diabetes and cardiovascular disease.

Methods: Principal component analysis (PCA) and Cronbach alpha assessed the reliability of the Attitudes about Complex Therapy Scale (ACTS). Examinations of relationships with related measures inform concurrent validity. Questionnaires were sent to a cross-sectional sample of 480 people prescribed multiple medicines for co-morbid Type 2 diabetes.

Results: Cronbach alpha was 0.76 , indicating the scale had good internal reliability. PCA rotated a four factor model accounting for $37 \%$ of the variance. Four subscales identified; 1 . Concerns about multiple medicines and increasing numbers of medicines; 2.Anxiety over missed medicines; 3. Desires to substitute medicines and reduce the number of medicines prescribed and; 4. Perceptions related to organising and managing complex therapy. The ACTS showed significant relationships with measures of anxiety, depression, general beliefs about medicines and self-efficacy. Also, the ACTS significantly correlated with adherence to medicines, showing good predictive validity.

Conclusion: The ACTS was designed to assess negative attitudes towards complex therapy and multiple medication management. This tool could aid prescribing decisions and may identify people who are intentionally non-adherent to all or some of their medicines.

Keywords: Adherence; Polypharmacy; Comorbid Diabetes; Cardiovascular Disease; Multiple Medicines.

\section{*Corresponding Author:}

Dr. Rebecca Stack,

Division of Psychology, School of Social Sciences, Nottingham Trent University, England.

Tel: 07944505686

E-mail: rebecca.stack@ntu.ac.uk

Received: September 30, 2015

Accepted: November 06, 2015

Published: November 12, 2015

Citation: Rebecca J Stack, et al., (2015) The Attitudes about Complex Therapy Scale (ACTS) in Type 2 Diabetes and Cardiovascular Disease: Development, Validity and Reliability. Int J Diabetol Vasc Dis Res, 3(9) 130136. doi: http://dx.doi.org/10.19070/2328-353X-1500028

Copyright: Rebecca J Stack ${ }^{\mathcal{O}} 2015$. This is an open-access article distributed under the terms of the Creative Commons Attribution License, which permits unrestricted use, distribution and reproduction in any medium, provided the original author and source are credited.

\section{Introduction}

Type 2 Diabetes (T2D) most often occurs not as a single impairment or risk factor, but as co-morbid with cardiovascular disease (CVD) and macrovascular complications [1-3]. Consequently patients diagnosed with T2D maybe required to manage medicines for hyperglycemia, hypertension and hyperlipidaemia [4]. Furthermore, clinical trials such as the United Kingdom Prospective Diabetes Study [5], Steno-2 Study [6] and Collaborative Atorvastatin Diabetes Study [7] advocate the intensification of medicines to improve long-term outcomes for patients. In addition to the management of multiple medicines, people with co-morbid T2D and CVD are often required to undertake lifestyle changes and perform a range of self-care activities including self-monitoring of blood pressure and blood glucose. The self-management of co-morbid T2D can be increasingly complex for patients.

Multiple barriers to medication adherence (the extent to which a patient takes their medicines as prescribed) in T2D have identified, including the cost of medicines [8], social barriers [9], and beliefs about illness and treatment [10]. In addition, the presence of co-morbidities and the prescription of multiple medicines may have a negative impact on the way people self-manage [11]. Studies have shown that patients prescribed more medicines (i.e. multiple daily doses) have lower rate of medication adherence $[12,13]$. Evidence suggests that increasing numbers of medicines leads to patient dissatisfaction and non-adherence [14].

A study of multiple medicine adherence in people taking multiple medicines for comorbid T2D found that when large numbers of medicines were prescribed, medicines for hyperlipidaemia were 
less likely to be taken (compared with medicines for hypertension and hypoglycaemia) [14]. Qualitative research has also found that CVD medicines are often undervalued by people with co-morbid T2D [15]. Patients explaining why low priority was given to CVD said they believed that CVD should be managed through lifestyle changes (not medicines) while T2D was perceived a severe condition requiring pharmacological management. Other studies have shown that patients with comorbid T2D tend prioritize their diabetes, and will adjusting their regimen to match their beliefs [16, 17]. Elliott et al. suggested that variable use of medicines, i.e. using some medicines more than others, is related to patients' personal prioritisation of medicines [18], while other studies have shown that multiple medicine regimens are associated with fear, uncertainty and lower control [19]. Also, patients fear chemical interactions and view medicines as a 'last resort', suggesting that people prescribed multiple medicines hold perceptions which reflect their personal self-management difficulties.

The beliefs people managing multiple medicines for T2D and CVD hold are not typically represented in measures of illness perceptions, treatment perceptions or self-management behaviours. Questionnaires such as the illness perception questionnaire [20] and the beliefs about medicines questionnaire (BMQ) [21] typically measure the perceptions of people with single illnesses receiving single treatments. Therefore, perceptions specifically related to the decisions faced by people managing multiple chronic conditions, such as the prioritisation of medicines, variable use of medicines and fears of chemical interactions are not yet captured in clinical or research measures. The development of a tool to measure perceptions relating to multiple medicines would help to identify the behavioural outcomes of negative perceptions towards multiple medicines and may also be of use to clinicians wishing to identify people who are experiencing difficulties in medicines management.

\section{Aim}

The aim of this research was to develop, validate and reliability test a new measure of patient perceptions of multiple medicines and difficulties in T2D and CVD. The attitudes about complex therapies scale (ACTS) was developed to address this aim.

\section{Method}

Ethics approval was granted from a Research Ethics Committee (reference number 07/Q1410/280).

\section{Participants}

Eligible participants were community dwelling adults (aged $>18$ years) managing multiple medicines for co-morbid T2D and CVD that is, prescribed one or more oral hypoglycaemic agents for the treatment of T2D; one or more anti-hypertensive drugs, and a statin for the treatment of hyperlipidaemia (see Table 1). The details of potential participants meeting the inclusion criteria were extracted from the secondary care diabetes database. Eligible participants had attended a secondary care diabetes clinic within 12 months of recruitment, to ensure that invitations were sent only to those who had recent healthcare services contact, and a medication review. For ethical reasons data could not be collected on non-participants.

\section{Procedure}

Participants were sampled for a secondary care diabetes database which incorporated prescribing information from secondary and primary care; this enhanced the reliability of the prescribing data used in this research. Eligible patients were sent a battery of questionnaires, an information sheet and letter of invitation plus free post return envelope. Participation was anonymous. Two reminders were sent to increase the response rate, the first two weeks after the initial mailing and the second followed two weeks after the first reminder. Informed signed consent was obtained from all participants.

\section{Measures}

The ACTS was developed from themes generated by 19 qualitative interviews with people managing multiple medicines for T2D and CVD. Sections of speech were taken from the verbatim interview transcripts, interpreted and converted into attitudes (a judgement about or preference towards an object, which is assumed to be driven by an underlying belief) and used as an item statement

Table 1. Number and percentage of participants prescribed each medicine.

\begin{tabular}{|c|c|}
\hline Name of medication & $\begin{array}{c}\text { Number of patients } \\
\text { prescribed medication (\%) }\end{array}$ \\
\hline Metformin & $388(80.33)$ \\
\hline Glitazones & $74(15.42)$ \\
\hline Sulphonylureas & $0(0)$ \\
\hline ACE Inhibitors & $324(67.5)$ \\
\hline Calcium channel blockers & $211(43.96)$ \\
\hline Angiotensin 2 receptor antagonists & $128(26.67)$ \\
\hline Diuretics & $126(26.25)$ \\
\hline Alpha blockers & $49(10.21)$ \\
\hline Beta Blockers & $0(0)$ \\
\hline Statins & $480(100)$ \\
\hline
\end{tabular}


for the ACTS. The interviews generated the following themes: Desires to avoid additional medicines; variable use of prescribed medicines; lifestyle modification vs. medicine use; chemicals vs. natural medicines; routine schedules and management; medication confusion and medication anxiety and emotions [15]. Thirtyfour items were extracted from patients statements (see Table 2), where possible the terminology and statement of the person interviewed was retained to generate scale times.

Where appropriate, items were reversed to prevent response bias. This was not always appropriate as sometimes positive wording detracted from its meaning, for example, "I don't ask the pharmacist to give me all the items on my prescription - I just don't need them all" could have been changed to "I ask the pharmacist to give me all the items on my prescription as they are all important" however this conveys a different type of attitude, behaviour and meaning. Only seven statements where the items were easily converted in to positive statements without losing meaning were selected.

Each item had a five point Likert scale response format, ranging from strongly disagree (valued at 1) through to a neutral response (valued at 3) through to strongly agree (valued at 5). Respondents indicated the degree of agreement with ACTS statements. The summation of the scores given to each item used indicates the level of negative attitudes towards multiple medicines.
The ACTS and five criterion measures formed a questionnaire battery which was sent to each eligible participant. The first two criterion measures were the general harm and general overuse scale of the BMQ [21]. Each BMQ general subscale contained four items, each measured on a five point Likert scale (score on each general subscale ranged between four and twenty). The third and fourth criterion measures were the depression and anxiety subscales of the Hospital Anxiety and Depression Scale (HADS) [22]. The HADS contains fourteen items and is a standard screening tool for depression (seven items) and anxiety (seven items) in healthcare setting. Each item is measured on a four point Likert scale; scores on the individual subscales range between zero and twenty-eight. The fifth criterion measure was the Diabetes Mellitus Self-efficacy (DMSE) scale [23]. The DMSE scale measures self-efficacy (motivation and perceived ability to perform a task) for tasks related to diabetes self-management. The scale consists of eight items on diet, exercise and medication management selfefficacy. Each item is measured on a ten point Likert scale; scores range between zero and eighty. The final measure used was the Morisky adherence scale [24]. This scale was used to explore the basic predictive validity of the ACTS tool.

\section{Analysis}

Principal component analysis (PCA) identified items that achieve higher order correlations that cluster theoretically and statistically to form factors (missing values were excluded pairwise). Theo-

Table 2. Items grouped according to the four factor rotation and factor loadings.

\begin{tabular}{|c|c|}
\hline Factor 1. Concerns about multiple medicines and increasing numbers of medicines & \\
\hline The chemical in my medicines may be damaging my body & .71 \\
\hline I'm scared the chemical in my medicines will interact with each other & .71 \\
\hline I don't know how my medicines work - it confuses me & .7 \\
\hline I'm scared of more medicines being added in the future & .6 \\
\hline I feel like more and more medicines are being added to my treatment plan & .57 \\
\hline I think some of my medicines should be taken away & .52 \\
\hline There is a particular medicines that I dislike above all the others I am taking & .52 \\
\hline Factor 2. Anxiety over missed medicines & \\
\hline Being put in a situation where I can't take my medicines worries me & .81 \\
\hline I become anxious if I miss my medication & .76 \\
\hline I feel guilty if I miss a dose or two & .75 \\
\hline Factor 3. Desire to substitute medicines and reduce the number of medicines prescribed & \\
\hline I plan to do more exercise to reduce the number of medicines I am taking & .77 \\
\hline I plan to improve my diet in order to reduce the number of medicines I am taking & .71 \\
\hline It may be possible for me to change my lifestyle and get rid of some of my medicines & .7 \\
\hline I would rather change something about my lifestyle than take extra medicines & .58 \\
\hline I plan to reduce the number of medicines I am taking & .55 \\
\hline In the future I would like to reduce the number of medicines I am taking & .45 \\
\hline I take most of my medicines regularly, but not all of them & .33 \\
\hline Factor 4. Organising and managing complex therapy & \\
\hline I'm not bothered by the number of medicines I've been prescribed I just take them REVERSE & .68 \\
\hline My medicines work because they are linked together and help each other out REVERSE & .49 \\
\hline I plan to take herbal medicines in the future & .44 \\
\hline I'm happy to be prescribed more medicines & .36 \\
\hline
\end{tabular}


retically, the ACTS items were likely to be correlated; therefore, an oblique direct oblimin rotation was undertaken. Concurrent validity assessed the relationship between the ACTS and tools which measure similar constructs or criterion measures. Concurrent validity was determined by the correlations between the ACTS, BMQ general sub-scale, DMSE and HADS. Cronbach's alpha assessed the internal consistency and identified items with poor alpha values. Multiple regression analyses using five predictor variables was conducted with scores on the 22 item ACTS. The predictor variables were the HADS anxiety, HADS depression, DMSE, BMQ harm and BMQ overuse. The relationship between scores on the Morisky adherence scale and the ACTS were examined.

\section{Results}

\section{Descriptive statistics}

Of the 878 questionnaires distributed, 23 were returned undelivered, 42 were returned unanswered. Of the 855 active sample participants, $480(56 \%)$ questionnaires were finally returned. Four hundred and eighty participants completed the 34-item ACTS. The sample size recommended for PCA is a minimum of 5, or 10-15 subjects per item $[25,26]$. The target sample size for the study was between 340 and 510, and was achieved.

The mean (SD, range) age of respondents was 66.31 years $(9.58$, 28-94); 308 respondents $(68.6 \%)$ were male. The mean score on the 34-item ACTS was $99.07(\mathrm{SD}=12.86)$ scores ranged between 50 and 140 and were normally distributed. Cronbach alpha for the ACTS was 0.78 ; which is generally accepted as indicating reliability. Table 1 shows the frequency of medicines prescribed. The mean number of medicines prescribed was 4.8 (range 3-12).

\section{Principle Component Analysis}

Preliminary assessment of the data was undertaken to determine its suitability for PCA. Two, four, five, six and seven factor rotations were inspected; the logical and theoretically soundness of each rotation was considered. Strong consideration was given to a six and a four factor solution; the four factor solution was accepted based on the break in the screen plot (see Figure 1 \& 2), and the component matrix (Table 2). The accumulative variance accounted by a four factor rotation explained $36.74 \%$ of the variance.

The four factor solution was rotated using a direct oblique PCA method. The factor loadings on each component were examined.
Items were deleted from the ACTS if the factor loading was less than 0.33 [27]. Also, items loaded on to a second factor with a factor loading of above 0.4 were also deleted. Following the deletion of items confirmatory PCA was undertaken to confirm the 4 factor solution. Table 2 shows the remaining 22 items in the ACTS following the four factor rotation.

\section{Cronbach alpha}

Cronbach alpha was calculated for the 22 item ACTS (following PCA). A good level of reliability for the 22 item ACTS was found (0.76). The overall internal consistency of each scale (as indicated by Cronbach's alpha values) was not improved by the deletion of any scale items. Cronbach alpha for the four factors rotated using PCA are as follows: factor $1=0.78$; factor $2=0.72$; factor $3=0.73$; factor $4=0.45$.

\section{Validity Testing}

The regression analysis revealed that the independent variables accounted for $39.7 \%$ of the variance in the ACTS (compared with $32.5 \%$ of the variance before PCA was undertaken and poorly performing items were removed). All criterion variables, with the exception of depression, were significant predictors of the ACTS. High scores on the ACTS were related to high scores on the HADS anxiety (beta $=0.16, \mathrm{p}<.01$ ) BMQ over-prescribing (beta $=0.33, \mathrm{p}<.01)$ and BMQ harm subscales (beta $=.21, \mathrm{p}<.01$ ). Scores on the ACTS were not significantly related to HADS depression scores (beta $=0.09$, sig $=0.16$ ). Finally, high score on the ACTS were significantly related to low scores on DM self-efficacy (beta $=-0.151, \mathrm{p}<.01$ ); therefore, high ACTS scores were related to lower levels of self-efficacy. Overall scores on the ACTS were significantly correlated with non-adherence to medicines $(r=364$, $\mathrm{p}<.01)$.

\section{Validity of the ACTS subscales}

Factor 1; The mean score was 21.29 (sd=4.91). The criterion measures accounted for $46 \%$ of the variance in the concerns about multiple medicines and increasing numbers of medicines subscale. Measures of depression and perceptions of harm were found to be significant predictors of this subscale (depression beta $=.228, \mathrm{p}<.01$; harm beta $=.376, \mathrm{p}<.01$ ). The concerns about multiple medicines and increasing numbers of medicines subscale was significantly correlated with non-adherence to medicines $(\mathrm{r}=.312, \mathrm{p}<.01)$ (see Table 3).

Factor 2; The mean score on this subscale was $8.48(\mathrm{sd}=2.76)$.

Figure 1. Scree plot for original pool of items.

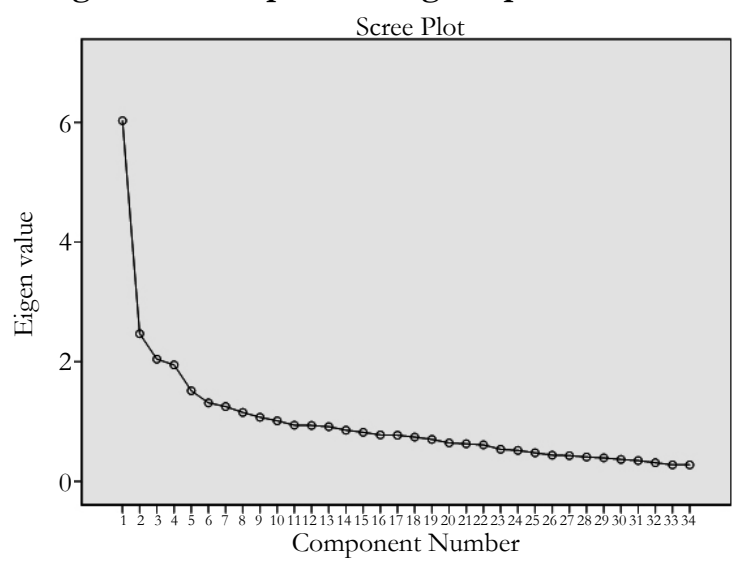


Figure 2. Scree plot following the deletion of items: 22 item ACTS.

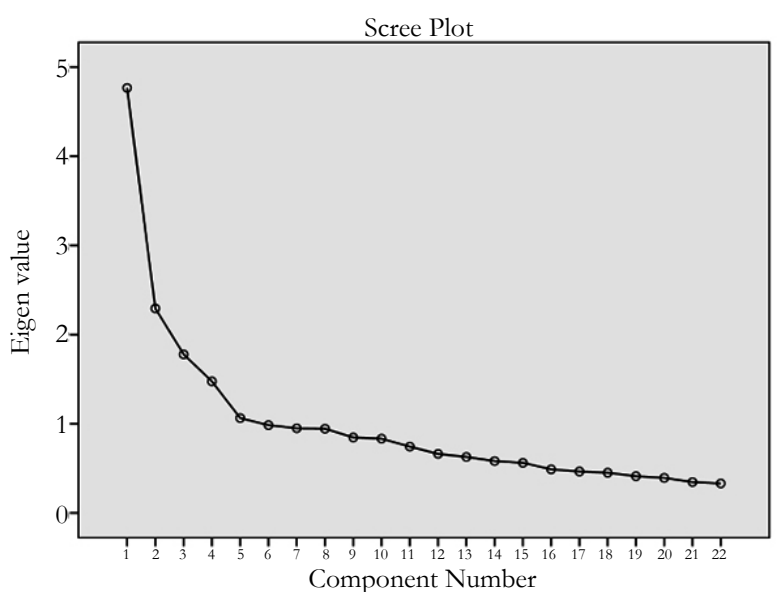

Table 3. Beta values and significance levels of the relationships between ACTS subscales and the criterion variables.

\begin{tabular}{|c|c|c|c|}
\hline & & Beta & Sig \\
\hline \multirow[t]{5}{*}{ 1. Factor 1} & HADS anxiety & .109 & .107 \\
\hline & HADS depression & .228 & .002 \\
\hline & DMSE (self-efficacy) & -.049 & .396 \\
\hline & BMQ over prescribing & .055 & .423 \\
\hline & BMQ harm & .376 & .000 \\
\hline \multirow[t]{5}{*}{ 2. Factor 2} & HADS anxiety & .256 & .000 \\
\hline & HADS depression & -.017 & .816 \\
\hline & DMSE (self-efficacy) & .042 & .481 \\
\hline & BMQ over prescribing & .011 & .848 \\
\hline & BMQ harm & -.008 & .901 \\
\hline \multirow[t]{5}{*}{ 3. Factor 3} & HADS anxiety & .111 & .13 \\
\hline & HADS depression & .211 & .005 \\
\hline & DMSE (self-efficacy) & -.096 & .089 \\
\hline & BMQ over prescribing & .325 & .000 \\
\hline & BMQ harm & .058 & .431 \\
\hline \multirow[t]{5}{*}{ 4. Factor 4} & HADS anxiety & .119 & .113 \\
\hline & HADS depression & -.052 & .492 \\
\hline & DMSE (self-efficacy) & -.063 & .247 \\
\hline & BMQ over prescribing & .234 & .002 \\
\hline & BMQ harm & .707 & .48 \\
\hline
\end{tabular}

The criterion measures accounted for $3.4 \%$ of the variance in the anxiety over missed medicines subscale. Anxiety was a significant predictor of this subscale (beta $=.256, \mathrm{p}<.01$ ). The anxiety over missed medicines subscale was not a significant predictor of nonadherence $(\mathrm{r}=.074, \mathrm{p}=.18)$.

Factor 3; The mean score was 22.35 ( $\mathrm{sd}=4.59$ ). The criterion measures accounted for $39.3 \%$ of the variance in the desire to substitute medicines and reduce the number of medicines prescribed subscale. Measure of depression and over-prescribing were significant predictors for this subscale (depression beta $=.221, \mathrm{p}<.01$; over-prescribing beta $=.234, \mathrm{p}<.01)$. The desire to substitute medicines and reduce the number of medicines prescribed subscale was significantly correlated with non-adherence to medicines $(\mathrm{r}=.233, \mathrm{p}<.01)$.

Factor 4; The mean score was 12.02 (sd=2.79). The criterion measures accounted for $22.2 \%$ of the variance in the perceptions related to organising and managing complex therapy subscale. The measure of perceptions of over prescribing was a significant predictor for this subscale (beta $=.234, \mathrm{p}<.01$ ). The perceptions related to organising and managing complex therapy subscale was significantly correlated with non-adherence $(r=.231, \mathrm{p}<.01)$.

\section{Discussion}

The ACTS was developed to measure attitudes towards taking multiple medicines for the management of T2D and CVD. The scale was originally developed from qualitative interviews and represents the difficulties people report facing when managing complex pharmacological therapies for T2D and CVD. Reliability and validity techniques were then applied to the ACTS to enhance the psychometric properties of this scale. Four key factors were identified through PCA: concerns about multiple medicines and increasing numbers of medicines; anxiety over missed medicines; desire to substitute medicines and reduce the number of 
medicines prescribed; and perceptions related to organising and managing complex therapy. These factors were consistent with themes previously identified in qualitative investigations with people managing complex medication regimens $[12,13]$.

The ACTS subscales, while representing the beliefs of people diagnosed with T2D and CVD, were found to share properties with measures of medication beliefs and psychological wellbeing. The BMQ general beliefs scale contains items related to a person general orientation towards medicines, and specifically asks about beliefs related to harm and over-prescribing [28]. The ACTS subscale on concerns about multiple medicines and increasing numbers of medicines was only found to correlate with BMQ subscale related to perceptions of medicines harm. Surprisingly, this subscale did not relate to the BMQ subscale on over prescribing. In addition increased concerns about multiple medicines and increasing numbers of medicines were correlated with depressive thoughts as measured by the HADS. Similarly to the self-regulation model and necessity-concern framework, we found emotion plays an important role in the management of multiple medicines. The ACTS represents emotional representation of medicine taking, as a subscale which focuses on feelings of anxiety or worry when medicines are forgotten or accidentally missed was identified. Higher scores on this subscale were associated with anxious thoughts (as measured by the HADS anxiety scale).

Unlike other beliefs scales, the ACTS, by focusing on the medication perceptions of a specific population, has identified unique characteristics such as the desires to reduce the number of medicines prescribed and substitute medicines with lifestyle interventions. Further research could explore whether patients who hold these beliefs are more motivated to engage in life-style changes; this subscale was significantly correlated with depression. It is possible that those who want to reduce or substitute their medicines may experience lower levels of perceived control and experience higher levels of depression. Other research has found a relationship between depression and non-adherence, and reported that this relationship was mediated by perceived control [29]. However, these relationships need to be explored in more depth before conclusions can be drawn.

The large dataset drawing participants from an integrated primary and secondary care settings and the good questionnaire response rate are methodological strengths of this research. Despite the benefits of this research, limitations of the ACTS should be acknowledged. A difficulty with PCA is that the names given to the factors rotated are subjectively derived. If a factor's label does not reflect the attributes that link items together, the rotation of a four-factor solution could be questioned. This issue could be resolved by undertaking confirmatory PCA on an alternative dataset to confirm the appropriateness of a four-factor solution. A disadvantage can arise within PCA if items share similar properties, but still remain fundamentally distinct from one another. In such cases, PCA may assign them to a single factor. However, it is widely accepted that the interpretation of PCA uses both objective and subjective criteria. In this case, it is the judgment of the Investigators that these items share a common theme that is reflected by the title given to the factor.

The choice was made to extract questionnaire items from qualitative interview findings, therefore, the scale items are statements made by people with T2D and CVD. In hindsight, further face validation of the questionnaire items in a larger population of people managing T2D and CVD, may have refine the phrasing of some questionnaire items. In addition, further work to ensure that scale items could be understood by people with lower levels of literacy is required. Future research should develop the ACTS through methods such as cognitive interviewing and Rasch modelling methods. These methods can be used to refine times, enhance validity and provide the ACTS with appropriate weights to the questionnaire items. The ability of the ACTS to predict nonadherence is shared with other measures. Further validation of this tool is required to demonstrate its distinctiveness from other scales especially the BMQ. There is some variation in the number of items within each scale which may have implications for the way the ACTS is scored. Balancing the contribution of each subscale to the overall score of the ACTS is important, and further investigation in to the weighting items is required.

The identification of non-adherence in people with T2D has been identified as a priority [30]. Scores on the ACTS were correlated with non-adherence to medicines, and identified specific medicine taking beliefs held by people with T2D. This tool could be used to identify people experiencing difficulties managing multiple medicines, and who are likely to be non-adherent to one of more of their medicines. The clinical use of the ACTS should be established, we encourage the use of the ACTS during consultations with patients prescribed multiple medicines for T2D and CVD.

\section{References}

[1]. Fortin M, Soubhi H, Hudon C, Bayliss EA, van den Akker M (2007) Multimorbidity's many challenges. BMJ 334(7602): 1016-1017.

[2]. Booth GL, Kapral MK, Fung K, Tu JV (2006) Recent trends in cardiovascular complications among men and women with and without diabetes. Diabetes Care 29(1): 32-37.

[3]. Booth GL, Kapral MK, Fung K, Tu JV (2006) Relation between age and cardiovascular disease in men and women with diabetes compared with non-diabetic people: a population-based retrospective cohort study. Lancet 368(9529): 29-36.

[4]. Kirsh SR, Aron DC (2011) Choosing targets for glycaemia, blood pressure and low-density lipoprotein cholesterol in elderly individuals with diabetes mellitus. Drugs Aging 28(12): 945-960.

[5]. Stratton IM, Cull CA, Adler AI, Matthews DR, Neil HA, et al. (2006) Additive effects of glycaemia and blood pressure exposure on risk of complications in type 2 diabetes: a prospective observational study (UKPDS 75). Diabetologia 49(8): 1761-1769.

[6]. Gaede P, Lund-Andersen H, Parving HH, Pedersen O (2008) Effect of a multifactorial intervention on mortality in type 2 diabetes. N Engl J Med 358(6): 580-591.

[7]. Colhoun HM, Betteridge DJ, Durrington PN, Hitman GA, Neil HA, et al. (2004) Primary prevention of cardiovascular disease with atorvastatin in type 2 diabetes in the Collaborative Atorvastatin Diabetes Study (CARDS): multicentre randomised placebo-controlled trial. Lancet 364(9435): 685-696.

[8]. Mackey K, Parchman ML, Leykum LK, Lanham HJ, Noel PH, et al. (2012) Impact of the Chronic Care Model on medication adherence when patients perceive cost as a barrier. Prim Care Diabetes 6(2): 137-142.

[9]. Jenkins N, Hallowell N, Farmer AJ, Holman RR, Lawton J (2011) Participants' experiences of intensifying insulin therapy during the Treating to Target in Type 2 Diabetes (4-T) trial: qualitative interview study. Diabet Med 28(5): 543-548.

[10]. Farmer A, Kinmonth AL, Sutton S (2006) Measuring beliefs about taking hypoglycaemic medication among people with Type 2 diabetes. Diabet Med 23(3): 265-270.

[11]. Morrow AS, Haidet P, Skinner J, Naik AD (2008) Integrating diabetes selfmanagement with the health goals of older adults: a qualitative exploration. Patient Educ Couns 72(3): 418-423.

[12]. Iskedjian M, Einarson TR, MacKeigan LD, Shear N, Addis A, et al. (2002) Relationship between daily dose frequency and adherence to antihypertensive pharmacotherapy: evidence from a meta-analysis. Clin Ther 24(2): $302-$ 316.

[13]. White AJ, Kellar I, Prevost AT, Kinmonth AL, Sutton S, et al. (2012) Adherence to hypoglycaemic medication among people with type 2 diabetes in primary care. Prim Care Diabetes 6(1): 27-33. 
[14]. Stack RJ, Bundy CE, Elliott R, New JP, Gibson M, et al. (2010) Intentional and unintentional non-adherence in community dwelling people with type 2 diabetes: the effect of varying numbers of medicines. The British journal of Diabetes and Vascular Disease 10(3): 148-152.

[15]. Stack RJ, Elliott RA, Noyce PR, Bundy C (2008) A qualitative exploration of multiple medicines beliefs in co-morbid diabetes and cardiovascular disease. Diabet Med 25(10): 1204-1210.

[16]. Anthony H, Valinsky L, Inbar Z, Gabriel C, Varda S (2012) Perceptions of hypertension treatment among patients with and without diabetes. BMC Fam Pract 13: 24.

[17]. Stack RJ, Bundy C, Elliott RA, New JP, Gibson JM, et al. (2011) Patient perceptions of treatment and illness when prescribed multiple medicines for co-morbid type 2 diabetes. Diabetes Metab Syndr Obes 4: 127-135.

[18]. Elliott RA, Ross-Degnan D, Adams AS, Safran DG, Soumerai SB (2007) Strategies for coping in a complex world: adherence behavior among older adults with chronic illness. J Gen Intern Med 22(6): 805-810.

[19]. Townsend A, Hunt K, Wyke S (2003) Managing multiple morbidity in midlife: a qualitative study of attitudes to drug use. BMJ 327(7419): 837.

[20]. Weinman J, Petrie KJ, Moss-Morris R, Horne R (1996) The Illness Perception Questionnaire: A new method for assessing the cognitive representation of illness. Psychology and Health 11(3): 431-446.

[21]. Horne R, Weinman J, Hawkins M (1999) The beliefs about medicines questionnaire: the development and evaluation of a new method for assessing the cognitive representation of medication. Psychology and Health 14(1): 1-24.
[22]. Zigmond AS, Snaith RP (1983) The hospital anxiety and depression scale. Acta Psychiatr Scand 67(6): 361-370.

[23]. Stanford Patient Education Research Centre. Diabetes Self-Efficacy Scale. 2011. Ref Type: Online Source

[24]. Morisky DE, Green LW, Levine DM (1986) Concurrent and predictive validity of a self-reported measure of medication adherence. Med Care 24(1): 67-74.

[25]. Pett MA, Lackey NR, Sullivan JJ (2003) Making sense of factor analysis. The use of factor analysis for instrument development in health care research. Sage Publications.

[26]. Hatcher LA (1994) A step-by-step approach to using the SAS(R) system for factor analysis and structural equation modeling. SAS Institute, North Carolina, US

[27]. Kline P (1994) An Easy Guide to Factor Analysis. Routledge, London.

[28]. Horne R, Weinman J (1999) Patients' beliefs about prescribed medicines and their role in adherence to treatment in chronic physical illness. J Psychosom Res 47(6): 555-567.

[29]. DiMatteo MR, Lepper HS, Croghan TW (2000) Depression is a risk factor for noncompliance with medical treatment: meta-analysis of the effects of anxiety and depression on patient adherence. Arch Intern Med 160(14): 2101-2107.

[30]. National Institute for Health and Clinical Excellence (2009) Medicines adherence: Involving patients in decisions about prescribed medicines and supporting adherence. London. 